\title{
Quark and Gluon Orbital Angular Momentum and Spin in Hard Processes*
}

\author{
S. V. Bashinsky and R. L. Jaffe \\ Center for Theoretical Physics \\ Laboratory for Nuclear Science \\ and Department of Physics \\ Massachusetts Institute of Technology \\ Cambridge, Massachusetts 02139
}

(MIT-CTP-2733, hep-ph/9804397. April 1998)

\begin{abstract}
We suggest a method of constructing gauge invariant quark and gluon distributions that describe an abstract QCD observable and apply this method to analyze angular momentum of a hadron. In addition to the known quark and gluon polarized structure functions, we obtain gauge invariant distributions for quark and gluon orbital angular momenta, and consider some basic properties of these distributions and their moments.
\end{abstract}

Email addresses: sergei@mit.edu, jaffe@mit.edu; phone: (617)-253-4858; fax: (617)$253-8674$.

*This work is supported in part by funds provided by the U.S. Department of Energy (D.O.E.) under cooperative research agreement \#DF-FC02-94ER40818. 


\section{INTRODUCTION}

Since the initial realization that quark spin accounts for only a small fraction of the nucleon spin 1, theorists have struggled to define 6 and suggest ways to measure the other components of the angular momentum in QCD. At first even the meaning of the quark spin contribution, $\Sigma$, to the nucleon spin was the subject of considerable debate. Now it is gradually agreed that different interpretations of $\Sigma$ reduce to renormalization scheme dependence made particularly irksome by the role of the axial anomaly in this channel. The gluon spin fraction, $\Delta G$, seems to be well defined in the context of renormalization group improved parton models [3], where the polarized gluon structure function $\Delta g\left(x_{\mathrm{Bj}}, Q^{2}\right)$ has the meaning 45 of a scale dependent gluon spin distribution over the Bjorken variable $x_{\mathrm{Bj}}$ " so that

$$
\Delta G\left(Q^{2}\right)=\int \mathrm{d} x_{\mathrm{Bj}} \Delta g\left(x_{\mathrm{Bj}}, Q^{2}\right)
$$

Of course, $\Delta g$ and $\Delta G$ are physical, gauge invariant quantities and experiments are being pursued to measure them. Nevertheless, there exists no local gauge invariant operator that could represent the space density of the gluon spin, a fact that has complicated the description of the gluon spin fraction.

One may expect to incorporate the existing quark and gluon spin fractions into the nucleon angular momentum sum rule [6]

$$
\frac{1}{2} \Sigma+L_{q}+\Delta G+L_{g}=\frac{1}{2}
$$

However, the situation with respect to the quark and gluon orbital angular momenta $L_{q}$ and $L_{g}$ is less satisfactory. There are no generally agreed upon definitions of $L_{q}$ and $L_{g}$, and no associated gauge invariant definitions of their parton distributions 6 , $f_{L_{q}}\left(x_{\mathrm{Bj}}, Q^{2}\right)$ and $f_{L_{g}}\left(x_{\mathrm{Bj}}, Q^{2}\right)$. Some authors 10 use $L_{q}$ and $L_{g}$ given by the gauge variant decomposition of ref. [6], which appears in eq. (54) of our paper, in the gauge $A^{+}=0$, but alternative gauge fixing methods 610 might also be considered. Besides, $A^{+}=0$ does not completely fix the gauge, and $L_{q}$ or $L_{g}$ obtained this way are not separately invariant under the residual gauge transformation, that is not permissible for physical objects.

We give a positive resolution to these questions in Sec. 3 of this paper, where we obtain scale dependent but otherwise unambiguous distribution functions for every term in eq. (2) in a single framework. This will be contrasted with the conventional gauge invariant operator description 11] of angular momentum in a gauge theory, where the decomposition (2) and the polarized gluon distribution $\Delta g$ are obscure

1 As usual, $x_{\mathrm{Bj}} \equiv Q^{2} / 2 P \cdot q$ where $P$ is the nucleon 4 -momentum, $q$ is the momentum transfer to the nucleon, and $Q^{2}=-q^{2}$. 
and might even seem unphysical. But first we find it useful to develop a general approach to the construction of parton distributions associated with an arbitrary physical observable that may characterize quark and gluon fields. This approach is presented in the Sec. 2. The premises and results of Secs. 2 and 3 are summarized in the conclusion.

\section{STRUCTURE FUNCTIONS FOR A PHYSICAL OBSERVABLE}

We want to describe some additive physical quantity $\Gamma$ relative to a deep inelastic process, such as inclusive leptoproduction, where the Bjorken variable $x_{\mathrm{Bj}} \equiv Q^{2} / 2 P \cdot q$ is fixed by the kinematics of the experiment (scattering angles and energies) and the information about the hadron state is limited to forward hadron matrix elements of certain operators. In a naive free parton model and the Bjorken limit $q^{-} \rightarrow \infty$

$$
x_{\mathrm{Bj}}=\frac{p^{+}}{P^{+}},
$$

where $p^{+}$and $P^{+}$are the "+" components of the struck parton and the target momenta correspondingly. Therefore a similar experiment sensitive to $\Gamma$ would directly provide "the density of $\Gamma$ " carried by quark and gluon partons with the momentum $p^{+}=x_{\mathrm{Bj}} P^{+}$, rather than $\Gamma$ itself. With this in mind, we construct gauge and boost invariant expressions for such densities that we call quark and gluon $\Gamma$ distributions, $f_{\Gamma^{q}}\left(x_{\mathrm{Bj}}\right)$ and $f_{\Gamma^{g}}\left(x_{\mathrm{Bj}}\right)$. The observable $\Gamma$ can be defined by any hermitian operator constructed from quark and gluon fields or as a generator of some fields transformation. We do not associate $\Gamma$ with a specific experiment measuring it. Therefore, in this paper we will not give a universal answer to the question: In what experiment one should measure a general observable, but we show what knowledge about $\Gamma$ can in principle be obtained in a deep inelastic process.

In order to construct the parton distribution of $\Gamma$ in QCD we should make clear what a quark or gluon parton is in an interacting theory. The subtlety here is in the issue of gauge invariance: a pure quark field in one gauge is a superposition of quarks and gluons in another. Different ways of gluon field gauge fixing predetermine different decomposition of the coupled quark-gluon fields into quark and gluon degrees of freedom. Similarly, one can generalize a gauge variant nonlocal operator, obtained, for example, by Fock space decompositions of free fields, to more than one gauge invariant expressions, raising the problem of deciding which is the "true" one. We choose to define quark and gluon partons in fully interacting QCD such that their distributions in $x_{\mathrm{Bj}}$ are given by the unambiguous experimentally accessible structure functions $q\left(x_{\mathrm{Bj}}\right)$ and $g\left(x_{\mathrm{Bj}}\right)$. We find that this definition also reproduces the measurable polarized structure functions $\Delta q\left(x_{\mathrm{Bj}}\right)$ and $\Delta g\left(x_{\mathrm{Bj}}\right)$. 
The required definition of QCD partons is also very appealing from mathematical point of view. The "gauge covariant" quanta of quark and gluon fields with a definite momentum $p^{+}=x_{\mathrm{Bj}} P^{+}$are the eigenstates of the generators of the covariant translations in the $x^{-}$direction:

$$
\begin{aligned}
& T_{-}^{q}\left(a^{-}\right): \psi(x) \rightarrow U\left(x, x+a^{-}\right) \psi\left(x+a^{-}\right), \\
& T_{-}^{g}\left(a^{-}\right): D_{\lambda}(x) \rightarrow U\left(x, x+a^{-}\right) D_{\lambda}\left(x+a^{-}\right) U\left(x+a^{-}, x\right),
\end{aligned}
$$

where $x+a^{-}$is the shorthand for $\left(x^{+}, x^{-}+a^{-}, x^{1}, x^{2}\right), U\left(x, x+a^{-}\right)$and $U\left(x+a^{-}, x\right)$ are the Wilson lines along the straight path between $x$ and $x+a^{-}$,

$$
U\left(x, x+a^{-}\right) \equiv P \exp \left[i g \int_{x+a^{-}}^{x} d \xi^{-} A^{+}(\xi)\right]
$$

with a similar equation for $U\left(x+a^{-}, x\right)=U^{\dagger}\left(x, x+a^{-}\right)$, and $D_{\lambda}(x) \equiv \partial_{\lambda}-i g A_{\lambda}(x)$, so that the second line in eq. (四) defines a transformation of the gluon field $A_{\lambda}(x)$. The transformation $T_{-}^{q}$ commutes with $T_{-}^{g}$ and each of them forms a one-parameter Lie group:

$$
\begin{gathered}
{\left[T_{-}^{q}\left(a^{-}\right), T_{-}^{g}\left(b^{-}\right)\right]=0,} \\
T_{-}^{q}\left(a_{2}^{-}\right) T_{-}^{q}\left(a_{1}^{-}\right)=T_{-}^{q}\left(a_{1}^{-}+a_{2}^{-}\right), \\
T_{-}^{g}\left(b_{2}^{-}\right) T_{-}^{g}\left(b_{1}^{-}\right)=T_{-}^{g}\left(b_{1}^{-}+b_{2}^{-}\right) .
\end{gathered}
$$

These properties are easily established from the fact that $T_{-}^{g}\left(a^{-}\right)$leaves $A^{+}(x)$, and consequently the Wilson line (5), invariant, as evident from the infinitesimal form of the transformations (11)

$$
\begin{aligned}
& \delta_{-}^{q} \psi=a^{-} D_{-} \psi, \\
& \delta_{-}^{g} A_{\lambda}=a^{-} F_{-\lambda} \Rightarrow \delta_{-}^{g} A^{+}=0 \text {. }
\end{aligned}
$$

Obviously, the transformations $T_{-}^{q}$ and $T_{-}^{g}$, as defined by eq. (田) or eq. (7), commute with gauge transformations.

Action of both $T_{-}^{q}\left(a^{-}\right)$on the quark field and $T_{-}^{g}\left(a^{-}\right)$, with the same parameter $a^{-}$, on the gluon field yields ordinary translation of these fields by $a^{-}$plus an overall gauge transformation. This additional gauge transformation does not affect any gauge invariant observable, therefore the gauge covariant translation of every field in the system is physically equivalent to their usual translation. This is not true, however, when one considers the transformations (四) for quarks and gluons individually. For example, application of $T_{-}^{q}\left(a^{-}\right)$to the quark field $\psi(x)$ without changing the gauge field $A_{\lambda}(x)$ gives a physically different field configuration from what would be obtained by the usual translation $\psi(x) \rightarrow \psi\left(x+a^{-}\right)$, because a change of the $\psi(x)$ phase without a compensatory change in $A_{\lambda}(x)$ is not a symmetry of QCD.

The quark and gluon fields $\psi(x)$ and $A_{\lambda}(x)$ can be decomposed into a sum of one-dimensional irreducible representations of the groups $T_{-}^{q}$ and $T_{-}^{g}$, which are the eigenstates of the corresponding generators. We will see that this decomposition 
specifies the desired parton decomposition in an interacting theory. The irreducible representations of $T_{-}^{q}$ and $T_{-}^{g}$ become trivial in the light-cone gauge $A^{+}=0$ where these transformations coincide with ordinary translations. Let us denote fields in this gauge by the superscript ' $L C$ '. Then

$$
\begin{aligned}
& \delta_{-}^{q} \psi^{L C}=a^{-} \partial_{-} \psi^{L C} \\
& \delta_{-}^{g} A_{\lambda}^{L C}=a^{-} \partial_{-} A_{\lambda}^{L C}
\end{aligned} .
$$

and the decomposition that we are seeking for is simply

$$
\begin{aligned}
\psi^{L C}(x) & =\int \frac{\mathrm{d} k^{+}}{2 \pi} e^{-i k^{+} x^{-}} \tilde{\psi}\left(k^{+}, \tilde{x}\right), \\
A_{\lambda}^{L C}(x) & =\int \frac{\mathrm{d} k^{+}}{2 \pi} e^{-i k^{+} x^{-}} \tilde{A}_{\lambda}\left(k^{+}, \tilde{x}\right)
\end{aligned}
$$

where

$$
\tilde{x} \equiv\left(x^{+}, x^{1}, x^{2}\right)
$$

Since $T_{-}^{q}$ and $T_{-}^{g}$ commute with gauge transformation, their action on fields in an arbitrary gauge

$$
\psi(x)=e^{i \alpha(x)} \psi^{L C}(x) \quad, \quad A_{\lambda}(x)=e^{i \alpha(x)}\left(A_{\lambda}^{L C}(x)+\frac{i}{g} \partial_{\lambda}\right) e^{-i \alpha(x)},
$$

amounts to translation of the "physical" components $\psi^{L C}$ and $A_{\lambda}^{L C}$ according to eq. (8) with the unphysical phase $\alpha(x)$ left invariant:

$$
T_{-}^{q}\left(a^{-}\right), T_{-}^{g}\left(a^{-}\right): \alpha(x) \rightarrow \alpha(x) .
$$

Therefore, the "parton decomposition" of fields in an arbitrary gauge is simply obtained by a gauge transformation of eqs. (9) and (10). These equations can be viewed as transition from $x$ to $\left(k^{+}, \tilde{x}\right)$ representation which is more suitable for deep inelastic scattering, but this transition is done in a way respecting gauge invariance and coincides with brute-force Fourier transformation along $x^{-}$direction only in the specific gauge $A^{+}=0$.

Our use of the adjective "physical" for fields in the gauge $A^{+}=0$ has been somewhat premature. There remain gauge transformations, for which the phase $\alpha(x)$ in eq. (12) does not depend on $x^{-}$, that preserve the condition $A^{+}=A_{-}=0$. All true physical observables must be invariant under this residual gauge symmetry as well. The fields $\tilde{\psi}\left(k^{+}, \tilde{x}\right)$ and $\tilde{A}_{\lambda}\left(k^{+}, \tilde{x}\right)$ defined by eqs. (9) and (10) transform under it as

$$
\begin{aligned}
& \tilde{\psi}\left(k^{+}, \tilde{x}\right) \rightarrow e^{i \alpha(\tilde{x})} \tilde{\psi}\left(k^{+}, \tilde{x}\right) \\
& \tilde{A}_{\lambda}\left(k^{+}, \tilde{x}\right) \rightarrow e^{i \alpha(\tilde{x})}\left(\tilde{A}_{\lambda}\left(k^{+}, \tilde{x}\right)+\frac{2 \pi i \delta\left(k^{+}\right)}{g} \partial_{\lambda}\right) e^{-i \alpha(\tilde{x})} .
\end{aligned}
$$


Notice that the inhomogeneous term in the residual gauge transformation of $\tilde{A}_{\lambda}$ appears only at $k^{+}=0$. We find it useful to split the field $\tilde{A}_{\lambda}\left(k^{+}, \tilde{x}\right)$ into a part $\tilde{G}_{\lambda}\left(k^{+}, \tilde{x}\right)$, transforming homogeneously, and a gauge field of the residual gauge group, $\mathcal{A}_{\lambda}(\tilde{x})$ :

$$
\tilde{A}_{\lambda}\left(k^{+}, \tilde{x}\right) \equiv 2 \pi \delta\left(k^{+}\right) \mathcal{A}_{\lambda}(\tilde{x})+\tilde{G}_{\lambda}\left(k^{+}, \tilde{x}\right),
$$

so that under a residual gauge transformation

$$
\mathcal{A}_{\lambda}(\tilde{x}) \rightarrow e^{i \alpha(\tilde{x})}\left(\mathcal{A}_{\lambda}(\tilde{x})+\frac{i}{g} \partial_{\lambda}\right) e^{-i \alpha(\tilde{x})}, \quad \tilde{G}_{\lambda}\left(k^{+}, \tilde{x}\right) \rightarrow e^{i \alpha(\tilde{x})} \tilde{G}_{\lambda}\left(k^{+}, \tilde{x}\right) e^{-i \alpha(\tilde{x})} .
$$

The decomposition (15) becomes unambiguous if we require

$$
\left.\tilde{G}_{\lambda}\left(k^{+}, \tilde{x}\right)\right|_{k^{+}=0} \equiv 0 \text {. }
$$

Then combining eqs. (10) and (15) one obtains

$$
\mathcal{A}_{\lambda}(\tilde{x})=\frac{1}{\int \mathrm{d} x^{-}} \int \mathrm{d} x^{-} A_{\lambda}^{L C}(x) .
$$

The fields $\tilde{\psi}\left(k^{+}, \tilde{x}\right), \tilde{G}_{\lambda}\left(k^{+}, \tilde{x}\right)$, and the residual gauge field $\mathcal{A}_{\lambda}(\tilde{x})$ provide the basis in the field space that we use to construct gauge invariant quark and gluon distributions for physical observables.

We associate a physical quantity $\Gamma$ with the field transformation generated by the quantum operator corresponding to $\Gamma$. We do not insist on the invariance of the QCD action under this field transformation. If it is a symmetry of the theory then there exists a corresponding conserved Noether current, and its charge, which will be the operator of $\Gamma$, commutes with the Hamiltonian of the system. If it is not, the quantum expectation value of $\Gamma$ for a particular state is still a well defined quantity, and spin or orbital angular momentum, which are not separately conserved in a relativistic system, provide such examples.

Of the possible choices of $\Gamma$, only those that commute with the parton-defining transformations $T_{-}^{q}$ and $T_{-}^{g}$ can be interpreted as physical properties of quarks and gluons distributed in $x_{\mathrm{Bj}}$ - that is, as "parton distributions of $\Gamma$ ". Thus we restrict ourselves to $\Gamma^{\prime}$ 's that commute with $T_{-}^{q}$ and $T_{-}^{g}$ :

$$
\left[\delta_{-}^{q}, \delta_{\Gamma}\right]=\left[\delta_{-}^{g}, \delta_{\Gamma}\right]=0 .
$$

This allows complete description of $\Gamma$ in a hadron in terms of its distributions over partons, $f_{\Gamma^{q, g}}\left(x_{\mathrm{Bj}}\right)$.

These distributions, i.e. parton densities of $\Gamma$, are easily constructed using the following trick: We apply $\Gamma$ independently to each subspace of fields with a definite $k^{+}$. Remembering that by eq. (19) $\Gamma$ can be diagonalized in the basis of $T_{-}^{q}$ and $T_{-}^{g}$ eigenfunctions, we consider the set of independent transformations labeled by a continuous parameter $x_{\mathrm{Bj}}$ : 


$$
\begin{aligned}
\delta_{\Gamma^{q}}^{\left(x_{\mathrm{Bj}}\right)} \tilde{\psi}\left(k^{+}\right) & =\left.i \epsilon \Gamma^{q} \tilde{\psi}\left(p^{+}\right) \delta\left(\frac{k^{+}-p^{+}}{P^{+}}\right)\right|_{p^{+}=x_{\mathrm{Bj}} P^{+}} \\
\delta_{\Gamma^{g}}^{\left(x_{\mathrm{Bj}}\right)} \tilde{G}_{\lambda}\left(k^{+}\right) & =\left.i \epsilon \Gamma_{\lambda}^{g \chi} \tilde{G}_{\chi}\left(p^{+}\right) \delta\left(\frac{k^{+}-p^{+}}{P^{+}}\right)\right|_{p^{+}=x_{\mathrm{Bj}} P^{+}},
\end{aligned}
$$

and

$$
\delta_{\Gamma^{q, g}}^{\left(x_{\mathrm{Bj}}\right)} \mathcal{A}_{\lambda}=0
$$

where $\Gamma^{q}$ and $\Gamma^{g}$ are the generators of $\delta_{\Gamma}$ restricted to the fields $\tilde{\psi}\left(k^{+}, \tilde{x}\right)$ and $\tilde{G}_{\lambda}\left(k^{+}, \tilde{x}\right)$ with $k^{+}=x_{\mathrm{Bj}} P^{+}$. The generators $\Gamma^{q}$ and $\Gamma^{g}$ can be operators acting on spinor, Lorentz, color, etc. indices as well as on the coordinate $x^{\perp} \equiv\left(x^{1}, x^{2}\right)$. For simplicity we slightly limit our choice of $\Gamma$ further, assuming that $\Gamma^{q}$ and $\Gamma^{g}$ do not depend on the parameter $q^{+}$and do not involve the coordinate $x^{+}$or derivatives with respect to it. This is not absolutely necessary but it simplifies the following equations. We will not need such complications for any of the quantities that are required for angular momentum description.

The generators of the field transformations defined by eqs. (20) and (21) are associated with a physical observable which is their normalized expectation in a hadron state $|P\rangle$. These observables will be shown to be the $\Gamma$ parton distributions that we are looking for. Applying Fourier transform to eqs. (20) and (21), one finds the action of $\delta_{\Gamma}^{\left(x_{\mathrm{Bj}}\right)}$ on the conventional fields $\psi^{L C}(x)$ and $A_{\lambda}^{L C}(x)$ :

$$
\begin{gathered}
\delta_{\Gamma^{q}}^{\left(x_{\mathrm{Bj}}\right)} \psi^{L C}(x)=\frac{i \epsilon P^{+}}{2 \pi} \int \mathrm{d} \xi^{-} e^{i x_{\mathrm{Bj}} P^{+} \xi^{-}} \Gamma^{q} \psi^{L C}\left(x+\xi^{-}\right) \\
\delta_{\Gamma^{g}}^{\left(x_{\mathrm{Bj}}\right)} A_{\lambda}^{L C}(x)=\frac{i \epsilon P^{+}}{2 \pi} \int \mathrm{d} \xi^{-} e^{i x_{\mathrm{Bj}} P^{+} \xi^{-}} \Gamma_{\lambda}^{g \chi}\left(A_{\chi}^{L C}\left(x+\xi^{-}\right)-\mathcal{A}_{\chi}(\tilde{x})\right) .
\end{gathered}
$$

These transformations are generated by the light-front charges

$$
Q^{(+)} \equiv \int \mathrm{d}^{2} x^{\perp} \int \mathrm{d} x^{-} J^{+}(x)
$$

of the corresponding canonical currentst:

$$
\begin{gathered}
J_{\Gamma^{q}}^{\mu}\left(x ; x_{\mathrm{Bj}}\right)=\frac{P^{+}}{2 \pi} \int \mathrm{d} \xi^{-} e^{i x_{\mathrm{Bj}} P^{+} \xi^{-}} \bar{\psi}(x) \gamma^{\mu} \Gamma^{q} \psi\left(x+\xi^{-}\right) \\
J_{\Gamma^{g}}^{\mu}\left(x ; x_{\mathrm{Bj}}\right)=\frac{i P^{+}}{2 \pi} \int \mathrm{d} \xi^{-} e^{i x_{\mathrm{Bj}} P^{+} \xi^{-}} F^{\mu \lambda}(x) \Gamma_{\lambda}^{g \chi}\left[A_{\chi}\left(x+\xi^{-}\right)-\mathcal{A}_{\chi}(\tilde{x})\right] .
\end{gathered}
$$

${ }^{2}$ By the canonical current for a field transformation $\delta_{\Gamma} \Phi=i \epsilon \Gamma(\Phi)$ we mean

$$
J_{\Gamma}^{\mu} \equiv \sum_{\substack{\text { all } \\ \text { fields }}} \frac{\partial \mathcal{L}}{\partial\left(\partial_{\mu} \Phi\right)}(-i) \Gamma(\Phi) .
$$


It is necessary to use the light-front charges instead of the usual $Q=\int \mathrm{d}^{3} x J^{0}(x)$ because the transformations (22)-(23) are non-local in $x^{-}$. We can imagine boosting ourselves to the infinite momentum frame, $K^{\prime}$, where the target hadron has an infinitely large momentum in the $\hat{x}_{3}^{\prime}$ direction:

$$
P^{\prime}=\left(E_{P}^{\prime}, 0,0, P^{\prime 3}\right) \text { with } P^{\prime 3} \rightarrow+\infty
$$

In this limit the integration plane in

$$
Q^{\prime}=\int \mathrm{d}^{3} x^{\prime} J^{\prime 0}\left(x^{\prime}\right)
$$

"approaches" the $x^{-}$axis. On the other hand, at least for fields confined in a massive hadron,

$$
\lim _{P^{\prime 3} \rightarrow+\infty} Q^{\prime}=Q^{(+)}
$$

therefore $Q^{(+)}$is the correct generator. One may also check this using the light-cone formulation of a gauge theory 12. The light-front charge $Q^{(+)}$is invariant under boosts along $\hat{x}_{3}$ and can be evaluated in any frame in which the hadron has zero transverse momentum, $P^{1}=P^{2}=0$, including the rest frame. Notice that if the currents (25), (26) were conserved, their usual charges, $Q$, would be frame independent and equal to the light-front ones, $Q^{(+)}$.

Now we define the quark or gluon distribution functions for the physical observable $\Gamma$ in a covariantly normalized hadron state $|P\rangle,\left\langle P^{\prime} \mid P\right\rangle=2 E_{P}(2 \pi)^{3} \delta^{(3)}\left(\mathbf{P}^{\prime}-\mathbf{P}\right)$, as the normalized expectation

$$
\begin{aligned}
f_{\Gamma^{q, g}}\left(x_{\mathrm{Bj}}\right) \equiv \frac{1}{\langle P \mid P\rangle}\left\langle P\left|Q_{\Gamma^{q, g}}^{(+)}\left(x_{\mathrm{Bj}}\right)\right| P\right\rangle= \\
\quad=\frac{1}{2 P^{+}\left(\int \mathrm{d}^{2} x^{\perp} \int \mathrm{d} x^{-}\right)}\left\langle P\left|\int \mathrm{d}^{2} x^{\perp} \int \mathrm{d} x^{-} J_{\Gamma^{q, g}}^{+}\left(x ; x_{\mathrm{Bj}}\right)\right| P\right\rangle .
\end{aligned}
$$

The distributions are normalized so that $f_{\Gamma^{q, g}}\left(x_{\mathrm{Bj}}\right)=\Gamma_{P} \delta\left(1-x_{\mathrm{Bj}}\right)$ in a free parton model with $|P\rangle$ replaced by a quark or gluon parton which is an eigenstate of the transformation (20) with the eigenvalue $\Gamma_{P}$. With such normalization, the sum of the first moments of the quark and gluon $\Gamma$ distributions gives exactly the expectation value of the observable $\Gamma$ for the hadron state $|P\rangle$ in the infinite momentum frame:

$$
\int_{-1}^{1} \mathrm{~d} x_{\mathrm{Bj}}\left(f_{\Gamma^{q}}+f_{\Gamma^{g}}\right)=\frac{\left\langle P\left|Q_{\Gamma}^{(+)}\right| P\right\rangle}{\langle P \mid P\rangle}=\lim _{P^{\prime 3} \rightarrow \infty} \frac{\left\langle P^{\prime}\left|Q_{\Gamma}^{\prime}\right| P^{\prime}\right\rangle}{\left\langle P^{\prime} \mid P^{\prime}\right\rangle} .
$$

The objects defined by eq. (30) are Lorentz boost invariant, provided $\Gamma^{q}$ and $\Gamma^{g}$ transform as implied by their Lorentz and spinor indexes. They are invariant under residual gauge transformation if $\Gamma^{q}$ and $\Gamma^{g}$ transform homogeneously as: 


$$
\Gamma^{q} \rightarrow e^{i \alpha(\tilde{x})} \Gamma^{q} e^{-i \alpha(\tilde{x})}, \quad \Gamma^{g} \rightarrow e^{i \alpha(\tilde{x})} \Gamma^{g} e^{-i \alpha(\tilde{x})}
$$

Some examples are $\Gamma^{q}=1, \gamma^{\mu}, \partial_{\perp}-i g \mathcal{A}_{\perp}$ and $\Gamma_{\lambda}^{g \chi}=\delta_{\lambda}^{\chi}$; we will have more examples in Sec. III . When $f_{\Gamma^{q}}\left(x_{\mathrm{Bj}}\right)$ and $f_{\Gamma^{g}}\left(x_{\mathrm{Bj}}\right)$ are invariant under the residual gauge group, they are by construction the same in an arbitrary gauge. An explicitly gauge covariant expression for the distributions is straightforwardly obtained by inserting the Wilson lines $U\left(x, x+\xi^{-}\right)$into eqs. (25) and (26) as $\bar{\psi} \ldots \psi \rightarrow \bar{\psi} U \ldots \psi$, $F \ldots A \rightarrow \operatorname{Tr}\left[F U \ldots A U^{\dagger}\right]$. Even though the currents in eq. (30) are not necessarily conserved and the charge operator depends on $x^{+}$, the diagonal matrix element between 4-momentum eigenstates $|P\rangle$ are $x^{+}$independent.

The composite operators in eqs. (25) and (26) have ultraviolet divergences 13 , which arise in perturbation theory as infinite loop integrals over $k_{\perp}$ in the products of two fields at the same $x^{\perp}$. These divergencies are regulated by a cutoff at $k_{\perp}^{2} \sim$ $Q^{2} \equiv-q^{2}$ leading to factors like $\alpha\left(\mu^{2}\right) \log \left(Q^{2} / \mu^{2}\right)$. In practice they are handled with standard methods of operator renormalization. Renormalization of objects similar to eq. (30) is described in the work [3] by Collins and Soper.

For our purposes we take the state $|P\rangle$ in the definition of distribution functions to be the hadron helicity eigenstate with the maximal possible helicity, e.g. $+\frac{1}{2}$ for a nucleon. Of course, for spin independent structure functions one could equally well average over helicities. For some problems, for example transversity distributions 14, one must consider off-diagonal matrix elements between states with different helicities but the same 4-momentum $P$.

Notice that the very last term, $\mathcal{A}_{\lambda}(\tilde{x})$, in eq. (26) does not contribute to the $\Gamma$ distributions. Indeed, it produces the following term in $f_{\Gamma^{g}}\left(x_{\mathrm{Bj}}\right)$ :

$$
\left.f_{\Gamma^{g}}\left(x_{\mathrm{Bj}}\right)\right|_{\mathcal{A}}=-\frac{i \delta\left(x_{\mathrm{Bj}}\right)}{2 P^{+}} \frac{\left\langle P\left|\int \mathrm{d}^{2} x^{\perp} \int \mathrm{d} x^{-} \int \mathrm{d} y^{-} F^{+\lambda}(x) \Gamma_{\lambda}^{g \chi} A_{\chi}\left(y^{-}, \tilde{x}\right)\right| P\right\rangle}{\int \mathrm{d}^{2} x^{\perp}\left(\int \mathrm{d} x^{-}\right)^{2}},
$$

where eq. (18) was used. Because of the $x_{\mathrm{Bj}}$ delta-function, this term trivially vanishes for $x_{\mathrm{Bj}} \neq 0$. One might worry that its value at $x_{\mathrm{Bj}}=0$ could affect the first moment of $f_{\Gamma^{g}}\left(x_{\mathrm{Bj}}\right)$. In fact, it does not: In the $A^{+}=0$ gauge $F^{+\lambda}(x)=\partial_{-} A^{\lambda}(x)$ and $A^{\lambda}(x)$ remains finite when $x^{-} \rightarrow \pm \infty$, thus the expression (33) vanishes as an infinite volume average of the derivative of a bounded function 3 .

The familiar structure functions $q\left(x_{\mathrm{Bj}}\right), \Delta q\left(x_{\mathrm{Bj}}\right), g\left(x_{\mathrm{Bj}}\right), \Delta g\left(x_{\mathrm{Bj}}\right)$ are instantly reproduced from eq. (30). Indeed, the quark number and chirality are counted by $\Gamma^{q}=1$ and $\Gamma^{q}=\gamma^{5}$ correspondingly. Translating the current matrix elements to the origin and canceling out the volume factors in the numerator and denominator of eq. (30) we find

${ }^{3}$ In this discussion we treat gluon fields as if they where classical. Since eq. (33) deals with physical expectation values, the conclusion $\left.\Delta f_{\Gamma^{g}}\left(x_{\mathrm{Bj}}\right)\right|_{\mathcal{A}} \equiv 0$ should remain valid for the quantum case. 


$$
\begin{gathered}
\Gamma^{q}=1 \quad \Rightarrow \quad f_{q}\left(x_{\mathrm{Bj}}\right)=\frac{1}{2 \pi \sqrt{2}} \int \mathrm{d} \xi^{-} e^{i x_{\mathrm{Bj}} P^{+} \xi^{-}}\left\langle P\left|\psi_{+}^{\dagger}(0) \psi_{+}\left(\xi^{-}\right)\right| P\right\rangle, \\
\Gamma^{q}=\gamma^{5} \quad \Rightarrow \quad f_{\Delta q}\left(x_{\mathrm{Bj}}\right)=\frac{1}{2 \pi \sqrt{2}} \int \mathrm{d} \xi^{-} e^{i x_{\mathrm{Bj}} P^{+} \xi^{-}}\left\langle P\left|\psi_{+}^{\dagger}(0) \gamma^{5} \psi_{+}\left(\xi^{-}\right)\right| P\right\rangle
\end{gathered}
$$

that are recognizable 15 as unpolarized, $q_{a}\left(x_{\mathrm{Bj}}\right)$, and polarized, $\Delta q_{a}\left(x_{\mathrm{Bj}}\right)$, quark distributionsf. One can easily write down the corresponding distributions $q_{a}\left(x_{\mathrm{Bj}}\right)$ and $\Delta q_{a}\left(x_{\mathrm{Bj}}\right)$ for a particular flavor $a=u, d, \ldots$ complementing $\Gamma^{q}$ 's in eq. (35) with a flavor projector $P_{f f^{\prime}}^{(a)}=\delta_{f a} \delta_{f^{\prime} a}$.

Similarly we proceed with the gluon structure functions $g\left(x_{\mathrm{Bj}}\right)$ and $\Delta g\left(x_{\mathrm{Bj}}\right)$. For the first one $\Gamma_{\lambda}^{g \chi}=\delta_{\lambda}^{\chi}$ so that

$$
f_{g}\left(x_{\mathrm{Bj}}\right)=\frac{i}{4 \pi} \int \mathrm{d} \xi^{-} e^{i x_{\mathrm{Bj}} P^{+} \xi^{-}}\left\langle P\left|F^{+\lambda}(0) A_{\lambda}\left(\xi^{-}\right)\right| P\right\rangle .
$$

Substituting $e^{i x_{\mathrm{Bj}} P^{+} \xi^{-}}$by $\left(i x_{\mathrm{Bj}} P^{+}\right)^{-1} \frac{\partial}{\partial \xi^{-}} e^{i x_{\mathrm{Bj}} P^{+} \xi^{-}}$, integrating by parts, and remembering that $\partial_{-} A_{\lambda}^{L C}=F_{\lambda}^{+L C}=-F_{\lambda}{ }^{+L C}$ we obtain

$$
f_{g}\left(x_{\mathrm{Bj}}\right)=\frac{1}{4 \pi x_{\mathrm{Bj}} P^{+}} \int \mathrm{d} \xi^{-} e^{i x_{\mathrm{Bj}} P^{+} \xi^{-}}\left\langle P\left|F^{+\lambda}(0) F_{\lambda}^{+}\left(\xi^{-}\right)\right| P\right\rangle+\left[\left.(\ldots)\right|_{\xi^{-}=-\infty} ^{\xi^{-}=+\infty}\right] .
$$

The first term on the right hand side of eq. (37) is a standard form of the unpolarized gluon distribution $g\left(x_{\mathrm{Bj}}\right)$ 15. It has been argued 过 that the second, surface, term in eq. (37) can be dropped. We postpone the discussion of the gluon spin dependent distribution $\Delta g\left(x_{\mathrm{Bj}}\right)$ until the next section, where it appears in a natural way.

\section{ANGULAR MOMENTUM IN DEEP INELASTIC SCATTERING}

The application of our formalism for obtaining $x_{\mathrm{Bj}}$-distributions that describe angular momentum in a hadron is straightforward: one should identify the corresponding transformations of the quark and gluon fields $\tilde{\psi}$ and $\tilde{G}$ and substitute their generators $\Gamma^{q}$ and $\Gamma^{g}$ into the master formulas (30). As mentioned earlier, we are interested in transformations that are diagonalized by the "parton decomposition" (9), (10) or equivalently that commute with the gauge covariant translations given by eq. (四). Among three-dimensional rotations these are the rotations about the $x^{3}$-axis generated by $J^{12}$ that transform the fields $\tilde{\psi}\left(p^{+}, \tilde{x}\right)$ and $\tilde{G}_{\lambda}\left(p^{+}, \tilde{x}\right)$ as

$$
\begin{gathered}
\delta_{J} \tilde{\psi}=-\epsilon\left[\left\{-\frac{i}{2} \sigma^{12}\right\}+\left\{x^{1} \partial_{2}-x^{2} \partial_{1}\right\}\right] \tilde{\psi}, \\
\delta_{J} \tilde{G}_{\lambda}=-\epsilon\left[\left\{-\left(\delta_{\lambda}^{1} \delta_{2}^{\chi}-\delta_{\lambda}^{2} \delta_{1}^{\chi}\right)\right\}+\left\{x^{1} \partial_{2}-x^{2} \partial_{1}\right\}\right] \tilde{G}_{\lambda} .
\end{gathered}
$$

${ }^{4}$ In eq. (35) and later $\psi_{+} \equiv \frac{1}{2} \gamma^{-} \gamma^{+} \psi$ is the "good" component of the Dirac field $\psi$, which is the dynamical field in light-cone quantization $[2]$. 
We consider the transformations generated by each of the four terms surrounded by braces in eq. (38) independently of the others. The first generators in each of these equations are naturally associated with the field spins whereas the second are the standard generators of orbital angular momentum. In order obtain to gauge invariant structure functions we must maintain covariance with respect to the residual gauge transformations, in which the local gauge transformation parameter does not depend on $x^{-}$. This can be achieved replacing $\partial_{i}$ by the residual gauge covariant derivative

$$
\mathcal{D}_{i} \equiv \partial_{i}-i g \mathcal{A}_{i}
$$

Similar to the situation at the beginning of the previous section, the replacement (39) adds only an overall unphysical gauge transformation to the rotation (38) when $\delta_{J}$ applies to both quark and gluon fields with the same infinitesimal angle $\epsilon$. Also notice that by construction of $\mathcal{A}_{\lambda}$ in eq. (18) we have $\mathcal{D}_{i}=\partial_{i}$ in any gauge where $\lim _{x^{-} \rightarrow \pm \infty} A_{i}^{L C}(x)=0$.

Splitting the transformation (38) into the four independent ones as described, we define

$$
\begin{aligned}
& \Sigma: \delta_{\Sigma} \tilde{\psi} \equiv i \epsilon \sigma^{12} \tilde{\psi}=i \epsilon \gamma^{0} \gamma^{3} \gamma^{5} \tilde{\psi} ; \\
& L_{q}: \quad \delta_{L_{q}} \tilde{\psi} \equiv-\epsilon\left(x^{1} \mathcal{D}_{2}-x^{2} \mathcal{D}_{1}\right) \tilde{\psi} ; \\
& \Delta G: \delta_{\Delta G} \tilde{G}_{\lambda} \equiv \epsilon\left(\delta_{\lambda}^{1} \delta_{2}^{\chi}-\delta_{\lambda}^{2} \delta_{1}^{\chi}\right) \tilde{G}_{\chi}=\epsilon \varepsilon^{+-}{ }_{\lambda}{ }^{\chi} \tilde{G}_{\chi} \text {; } \\
& L_{g}: \delta_{L_{g}} \tilde{G}_{\lambda} \equiv-\epsilon\left(x^{1} \mathcal{D}_{2}-x^{2} \mathcal{D}_{1}\right) \tilde{G}_{\lambda} \text {. }
\end{aligned}
$$

The corresponding generators are

$$
\begin{array}{ll}
\Gamma_{\Sigma}=\sigma^{12}=\gamma^{0} \gamma^{3} \gamma^{5} & , \quad \Gamma_{L_{q}}=\left(x^{1} i \mathcal{D}_{2}-x^{2} i \mathcal{D}_{1}\right) \\
\left(\Gamma_{\Delta G}\right)_{\lambda} \chi=-i \varepsilon^{+-}{ }_{\lambda} \chi & ,
\end{array}
$$

where $\varepsilon^{\mu \nu \alpha \beta}$ is the antisymmetric Levi-Civita tensor with $\varepsilon^{0123} \equiv+1$ and $\varepsilon^{+-12}=-1$.

Following tradition the generator for $\Sigma$ is defined without the $\frac{1}{2}$ factor that appears in front of $\sigma^{12}$ in eq. (38). Substituting these $\Gamma$ 's into eqs. (25)-(26), (30) and remembering that the $\mathcal{A}_{\lambda}(\tilde{x})$ term in eq. (26) can be dropped, we obtain the following structure functions for the quark and gluon spins and angular momenta:f

${ }^{5}$ We remind the reader that $\psi_{+} \equiv \frac{1}{2} \gamma^{-} \gamma^{+} \psi$, the hadron $|P\rangle$ is polarized along its momentum $\mathbf{P}$, and the fields $\psi, A_{\lambda}$, and $F^{\mu \nu}$ in the structure functions below are either connected by a Wilson line to make these expressions gauge invariant or are evaluated in the gauge $A^{+}=0$, where the Wilson line is unity. 


$$
\begin{aligned}
f_{\Sigma}\left(x_{\mathrm{Bj}}\right) & =\frac{1}{2 \pi \sqrt{2}} \int \mathrm{d} \xi^{-} e^{i x_{\mathrm{Bj}} P^{+} \xi^{-}}\left\langle P\left|\psi_{+}^{\dagger}(0) \gamma^{5} \psi_{+}\left(\xi^{-}\right)\right| P\right\rangle ; \\
f_{L_{q}}\left(x_{\mathrm{Bj}}\right) & =\frac{\int \mathrm{d} \xi^{-} e^{i x_{\mathrm{Bj}} P^{+} \xi^{-}}\left\langle P\left|\int \mathrm{d}^{2} x^{\perp} \psi_{+}^{\dagger}\left(x^{\perp}\right)\left(x^{1} i \mathcal{D}_{2}-x^{2} i \mathcal{D}_{1}\right) \psi_{+}\left(x^{\perp}+\xi^{-}\right)\right| P\right\rangle}{2 \pi \sqrt{2}\left(\int \mathrm{d}^{2} x^{\perp}\right)} ; \\
f_{\Delta G}\left(x_{\mathrm{Bj}}\right) & =\frac{1}{4 \pi} \int \mathrm{d} \xi^{-} e^{i x_{\mathrm{Bj}} P^{+} \xi^{-}}\left\langle P\left|F^{+\lambda}(0) \varepsilon^{+-}{ }_{\lambda}{ }^{\chi} A_{\chi}\left(\xi^{-}\right)\right| P\right\rangle ; \\
f_{L_{g}}\left(x_{\mathrm{Bj}}\right) & =\frac{i \int \mathrm{d} \xi^{-} e^{i x_{\mathrm{Bj}} P^{+} \xi^{-}}\left\langle P\left|\int \mathrm{d}^{2} x^{\perp} F^{+\lambda}\left(x^{\perp}\right)\left(x^{1} i \mathcal{D}_{2}-x^{2} i \mathcal{D}_{1}\right) A_{\lambda}\left(x^{\perp}+\xi^{-}\right)\right| P\right\rangle}{4 \pi\left(\int \mathrm{d}^{2} x^{\perp}\right)} .
\end{aligned}
$$

In these expressions we have translated fields to the origin and canceled overall integrals where it is possible. One can replace the vector potential $A_{\lambda}$ in eqs. (44) and (45) in favor of the gluon field strength $F_{\lambda}{ }^{+}$as was done when we derived eq. (37). Dropping the surface terms, we obtain: (below $F^{*} \mu \nu \equiv \frac{1}{2} \varepsilon^{\mu \nu \alpha \beta} F_{\alpha \beta}$ )

$$
\begin{aligned}
f_{\Delta G}\left(x_{\mathrm{Bj}}\right) & =\frac{i}{4 \pi x_{\mathrm{Bj}} P^{+}} \int \mathrm{d} \xi^{-} e^{i x_{\mathrm{Bj}} P^{+} \xi^{-}}\left\langle P\left|F^{+\lambda}(0) F_{\lambda}^{*}{ }_{\lambda}^{+}\left(\xi^{-}\right)\right| P\right\rangle, \\
f_{L_{g}}\left(x_{\mathrm{Bj}}\right) & =\frac{\int \mathrm{d} \xi^{-} e^{i x_{\mathrm{Bj}} P^{+} \xi^{-}}\left\langle P\left|\int \mathrm{d}^{2} x^{\perp} F^{+\lambda}\left(x^{\perp}\right)\left(x^{1} i \mathcal{D}_{2}-x^{2} i \mathcal{D}_{1}\right) F_{\lambda}^{+}\left(x^{\perp}+\xi^{-}\right)\right| P\right\rangle}{4 \pi x_{\mathrm{Bj}} P^{+}\left(\int \mathrm{d}^{2} x^{\perp}\right)} .
\end{aligned}
$$

These are our principal results. Equations (42)-(47) give a complete set of distribution functions needed to describe a hadron's angular momentum. These expressions are automatically gauge and boost invariant as a consequence of our method. Our expressions for orbital angular momentum differ from the naive gauge invariant form in which $D_{i}$ appears instead of $\mathcal{D}_{i}$. This distinction is essential because $\mathcal{D}_{i}$ depends only on $\tilde{x}$ whereas $D_{i}$ is also a function of $x^{-}$, which is impossible to interpret in a parton model formalism. Our distribution functions can be written in an explicitly gauge-invariant form with use of the Wilson connection. We devote the rest of the section to analyzing these expressions and comparing them with earlier works.

In a non-interacting parton model the structure functions $f_{\Sigma}, f_{L_{q}}, f_{\Delta G}$, and $f_{L_{g}}$ describe the $x_{\mathrm{Bj}}$-distributions of spin and orbital angular momentum $x^{3}$-projections carried by quark and gluon partons respectively. This is also true in the interacting theory when one defines quark and gluon partons as excitations of the fields $\tilde{\psi}\left(p^{+}, \tilde{x}\right)$ and $\tilde{G}_{\lambda}\left(p^{+}, \tilde{x}\right)$ and their spin and orbital momentum as the charges of the transformations (40). We demonstrated that these are the definitions that reproduce the familiar unpolarized and polarized quark and gluon structure functions. According to eq. (31]), the first moments of the structure functions

$$
\Gamma \equiv \int_{-1}^{1} \mathrm{~d} x_{\mathrm{Bj}} f_{\Gamma}\left(x_{\mathrm{Bj}}\right)
$$


should give the total quark/gluon spin/orbital momentum in the hadron in the infinite momentum frame. The sum of the first moments obeys the angular momentum sum rule in eq. (2), where $1 / 2$ on the right hand side is the $x^{3}$-projection of the nucleon spin and should be replaced correspondingly for a hadron with a different spin. The first moment of $f_{\Sigma}$

$$
\Sigma=\frac{1}{\sqrt{2} P^{+}}\left\langle P\left|\psi_{+}^{\dagger}(0) \gamma^{5} \psi_{+}(0)\right| P\right\rangle
$$

indeed equals the total $x^{3}$-projection of the quark spin

$$
\Sigma\left(P^{\prime}\right)=\frac{\left\langle P^{\prime}\left|\int \mathrm{d}^{3} x \bar{\psi}(x) \gamma^{3} \gamma^{5} \psi(x)\right| P^{\prime}\right\rangle}{\left\langle P^{\prime} \mid P^{\prime}\right\rangle}=\frac{1}{2 E_{P}^{\prime}}\left\langle P^{\prime}\left|\bar{\psi}(0) \gamma^{3} \gamma^{5} \psi(0)\right| P^{\prime}\right\rangle
$$

as $P^{\prime 3} \rightarrow+\infty$.

The situation for $L_{q}, \Delta G$, and $L_{g}$ is a little more subtle. The first moments of the corresponding structure functions are

$$
\begin{aligned}
L_{q} & =\frac{1}{\sqrt{2} P^{+}\left(\int \mathrm{d}^{2} x^{\perp}\right)}\left\langle P\left|\int \mathrm{d}^{2} x^{\perp} \psi_{+}^{\dagger}\left(x^{\perp}\right)\left(x^{1} i \mathcal{D}_{2}-x^{2} i \mathcal{D}_{1}\right) \psi_{+}\left(x^{\perp}\right)\right| P\right\rangle, \\
\Delta G & =\frac{1}{2 P^{+}}\left\langle P\left|F^{+\lambda}(0) \varepsilon^{+-}{ }_{\lambda}^{\chi} A_{\chi}(0)\right| P\right\rangle \\
L_{g} & =\frac{i}{2 P^{+}\left(\int \mathrm{d}^{2} x^{\perp}\right)}\left\langle P\left|\int \mathrm{d}^{2} x^{\perp} F^{+\lambda}\left(x^{\perp}\right)\left(x^{1} i \mathcal{D}_{2}-x^{2} i \mathcal{D}_{1}\right) A_{\lambda}\left(x^{\perp}\right)\right| P\right\rangle .
\end{aligned}
$$

Up to the replacement $\partial_{i} \leftrightarrow \mathcal{D}_{i}$, the eqs. (49) and (51)-(53) reproduce the normalized forward matrix elements of the four operators in the gauge variant angular momentum decomposition by Jaffe and Manohar [6]

$$
J^{3}=\int \mathrm{d}^{3} x\left[\frac{1}{2} \bar{\psi} \gamma^{3} \gamma^{5} \psi+\psi^{\dagger}(\vec{x} \times i \vec{\partial})^{3} \psi+(\vec{E} \times \vec{A})^{3}-E^{k}(\vec{x} \times \vec{\partial})^{3} A^{k}\right]
$$

evaluated in the $A^{+}=0$ gauge and the infinite momentum frame. This result is quite welcome in view of previous studies [6] of angular momentum in deep inelastic scattering. This implies that many previous calculations, including $Q^{2}$ evolution and asymptotic behavior $8[0]$, obtained in $A^{+}=0$ gauge are transferable to our case with only minor modifications to incorporate $\partial_{i} \rightarrow \mathcal{D}_{i}$.

On the other hand, it is also known 11] that the total angular momentumf

$$
J^{k}=\frac{1}{2} \varepsilon^{k i j} \int \mathrm{d}^{3} x\left(x^{i} \Theta^{0 j}-x^{j} \Theta^{0 i}\right)
$$

${ }^{6}$ In the following eq. (55) $\Theta^{\mu \nu}$ stands for the symmetric energy-momentum Belifante tensor. 
can be decomposed into three gauge-invariant vectors: 11]

$$
\vec{J}=\int \mathrm{d}^{3} x\left[\frac{1}{2} \bar{\psi} \vec{\gamma} \gamma^{5} \psi+\psi^{\dagger}(\vec{x} \times i \vec{D}) \psi+\vec{x} \times(\vec{E} \times \vec{B})\right]
$$

where

$$
E^{k}=F^{k 0}, \quad B^{k}=-\frac{1}{2} \varepsilon^{k l m} F_{l m}
$$

are the color-electric and color-magnetic field vectors. The second term in eq. (56) is an alternative candidate for a definition of the quark orbital angular momentum. The third, gluon, term does not allow any further separation into local gauge-invariant operators that would describe spin and orbital momentum of gluons 7. The $d^{3} x$ integrals of each of the three terms in eq. (56) are still not covariant under Lorentz boosts so one have to specify a frame in which they are evaluated. These quantities are in principle measurable and can be related to form-factors of deeply virtual Compton scattering 16. Nevertheless, the decomposition (56) is not favorable for deep inelastic scattering experimental conditions in the sense that the separate terms in eq. (56) mix partons with different $p^{+}$and thus can not be presented in terms of parton densities. Of course the greatest shortcoming of the decomposition of eq. (56) is its inability to describe the gluon spin spin distribution $\Delta g\left(x_{\mathrm{Bj}}\right)$ which is clearly gauge invariant and can be measured in a variety of processes. There is no such a difficulty in the suggested approach where $\Delta g\left(x_{\mathrm{Bj}}\right)=f_{\Delta G}\left(x_{\mathrm{Bj}}\right)$.

\section{CONCLUSION}

Typically in (semi)-inclusive deep inelastic scattering the Bjorken variable $x_{\mathrm{Bj}}$ is fixed, and only forward hadron matrix elements are accessible. In order to accommodate these experimental conditions, we propose to describe a hadron in terms of those observables $\Gamma$ that are diagonal in the basis formed by quark and gluon partons. We then construct gauge invariant $x_{\mathrm{Bj}}$-distributions associated with such an observable. The gauge covariant definition of parton states in fully interacting QCD are chosen so that the parton distributions are given by the physical structure functions $q\left(x_{\mathrm{Bj}}\right)$ and $g\left(x_{\mathrm{Bj}}\right)$. To satisfy this requirement, quark and gluon partons must be eigenstates of the generators of covariant translations $T_{-}^{q}$ and $T_{-}^{g}$, eq. (田), and the $\Gamma$ distributions are given by the formulas

\footnotetext{
${ }^{7}$ We were able to give gauge invariant definitions for the gluon spin and angular momentum projections only on the axis $x^{3}$ defined by the external momentum $q$. It is not possible to define gauge invariant tensors of gluon spin and orbital momentum independent of a specified direction in space-time.
} 


$$
f_{\Gamma^{q}}\left(x_{\mathrm{Bj}}\right)=\frac{\left\langle P\left|\int \mathrm{d}^{2} x^{\perp} \int \mathrm{d} x^{-} \int \mathrm{d} \xi^{-} e^{i x_{\mathrm{Bj}} P^{+} \xi^{-}} \bar{\psi}(x) \gamma^{+} \Gamma^{q} \psi\left(x+\xi^{-}\right)\right| P\right\rangle}{4 \pi\left(\int \mathrm{d}^{2} x^{\perp} \int \mathrm{d} x^{-}\right)}
$$

and

$$
f_{\Gamma^{g}}\left(x_{\mathrm{Bj}}\right)=\frac{\left\langle P\left|\int \mathrm{d}^{2} x^{\perp} \int \mathrm{d} x^{-} \int \mathrm{d} \xi^{-} e^{i x_{\mathrm{Bj}} P^{+} \xi^{-}} F^{+\lambda}(x) \Gamma_{\lambda}^{g \chi} F_{\chi}^{+}\left(x+\xi^{-}\right)\right| P\right\rangle}{4 \pi x_{\mathrm{Bj}} P^{+}\left(\int \mathrm{d}^{2} x^{\perp} \int \mathrm{d} x^{-}\right)}
$$

in $A^{+}=0$ gauge. They may be put in a gauge-invariant form by trivial use of Wilson lines. In these formulas the generators $\Gamma^{q}$ and $\Gamma^{g}$ transform under the residual gauge group as given by eq. (32) and are uniquely related to the original quantity $\Gamma$.

A hadron angular momentum is completely described by the four scale dependent $x_{\mathrm{Bj}}$-distributions given by eqs. (42)-(43)), (46)-(47). Two of them, $f_{\Sigma}$ and $f_{\Delta G}$, coincide with the polarized quark and gluon structure functions $\Delta q$ and $\Delta g$. The other two are naturally regarded as the $x_{\mathrm{Bj}}$ distributions of quark and gluon orbital angular momentum. They are well defined physical objects and are gauge invariant by means of the residual gauge covariant derivative $\mathcal{D}_{i}=\partial_{i}-i g \mathcal{A}_{i}$, where $\mathcal{A}$ is given by eq. (18). They are observable in principle and can be calculated in models or within lattice QCD. However, it remains an open question whether the $x_{\mathrm{Bj}}$-distributions of quark and gluon orbital angular momentum which we have defined can be measured in a practical experimental process.

\section{ACKNOWLEDGMENTS}

We are grateful to J. Goldstone, D. Dolgov, J. Negele, and A. Pochinsky for helpful discussions. R.L.J. would also like to thank the RIKEN-BNL Research Center for partial support. 


\section{REFERENCES}

[1] J. Ashman et al., Phys. Lett. B 206, 364 (1988);

J. Ashman et al., Nucl. Phys. B 328, 1 (1988).

[2] P. G. Ratcliffe, Phys. Lett. B 192, 180 (1987).

[3] J. C. Collins and D. E. Soper, Nucl. Phys. B 194, 445 (1982).

[4] A. V. Manohar, Phys. Lett. B 255, 579 (1991);

I. I. Balitskii and V. M. Braun, Phys. Lett. B 267, 405 (1991).

[5] R. L. Jaffe, Phys. Lett. B 365, 359 (1996).

[6] R. L. Jaffe and A. Manohar, Nucl. Phys. B 337, 509 (1990).

[7] After the completion of this paper we became aware of a recent work by P. Hoodbhoy, X. Ji, and W. Lu that addresses some similar issues: hep-ph/9804337.

[8] X. Ji, J. Tang, and P. Hoodbhoy, Phys. Rev. Lett. 76, 740 (1996).

[9] P. Hägler and A. Schäfer, hep-ph/9802362.

[10] A. Harindranath, R. Kundu, hep-ph/9802406.

[11] X. Ji, Phys. Rev. Lett. 78, 610 (1997).

[12] J. B. Kogut and D. E. Soper, Phys. Rev. D 1, 2901 (1970).

[13] J. C. Collins, Phys. Rev. D 21, 2962 (1980).

[14] J. Ralston and D. E. Soper, Nucl. Phys. B 152, 109 (1979);

J. L. Cortes, B. Pire, and J. P. Ralston, Z. Phys. C 55, 409 (1992);

R. L. Jaffe and X. Ji, Phys. Rev. Lett. 67, 552 (1991); Nucl. Phys. B 375, 527 (1992).

[15] See, for example, R. L. Jaffe, Spin, Twist, and Hadron Structure in Deep Inelastic Processes, Lectures given at Ettore Majorana International School of Nucleon Structure (1995): hep-ph/9602236.

[16] X. Ji, Phys. Rev. D 55, 7114 (1997). 